\title{
Alcohol's harm to others: Using qualitative research to complement survey findings
}

\author{
Elizabeth Manton $^{1,2}$, Sarah MacLean ${ }^{1,3}$, Anne-Marie Laslett ${ }^{1,3}$, and Robin Room ${ }^{1,3}$ \\ ${ }^{1}$ Centre for Alcohol Policy Research, Turning Point, Fitzroy, Victoria, Australia \\ ${ }^{2}$ Eastern Health Clinical School, Faculty of Medicine, Nursing and Health Sciences, Monash University, Victoria, Australia \\ ${ }^{3}$ Centre for Health and Society, Melbourne School of Population and Global Health, The University of Melbourne, Victoria, Australia
}

\begin{abstract}
Aim: The purpose of this study was to identify the potential contribution of qualitative research to future Alcohol's Harm to Others (AHTO) survey research and some of the potential difficulties that may be encountered when conducting studies of this nature.
\end{abstract}

Design: Qualitative, in-depth semi-structured telephone interviews.

Setting: Australia.

Participants: Potential participants were those who responded, in the telephone land-line-based Australia-wide AHTO survey in either 2008 or 2011, that a child or children for whom they had responsibility had been harmed “a lot" or "a little” by someone else's drinking, and who also indicated that they were willing to be recontacted for future research interviews. Ten participants who selected the response "a lot" and 10 who selected "a little" were interviewed.

Measures: Interviews were audio recorded and professionally transcribed. Transcribed interviews were thematically analysed.

Findings: The qualitative study analysis enabled access to detailed stories, clarification of the validity and meanings of survey measures, identification of questions for future surveys, and contextualization of survey findings. The analysis also suggested that samples of people who agree to discuss harm from others' drinking with a researcher are likely to be skewed in particular ways.

Conclusions: The approach to AHTO research described here incorporates both the persuasive power of whole-population survey research and the nuanced understanding provided through interpretation of in-depth qualitative interviews. It enables the presentation of more comprehensive information about the nature and extent of AHTO.

Although the nature and extent of harm that drinkers do to themselves has been documented both in Australia and internationally, far less well understood is the harm that drinkers do to others as a result of their problematic drinking. In Australia, the first detailed study of harms from others' drinking at the population level, including harms in the household and family, among friends and coworkers, and from strangers on the street, was conducted in 2008 (Laslett et al., 2010). Australian alcohol's harm to others (AHTO) research has drawn wide international attention, and is currently being replicated in over a dozen countries, in studies tailored to fit local contexts. Recently, studies of AHTO have been identified by the World Health Organization (WHO) as a priority in its Global Strategy to Reduce Harmful Use of Alcohol (WHO, 2010), which aims to guide new interventions and galvanize public and legislative support for policies to reduce the social costs and harms of drinking (Giesbrecht, Cukier, \& Steeves, 2010). A study in seven low- and middle-income countries coordinated by the WHO and Thai Health includes population surveys and also scoping studies, in which key informants will be asked to share their understandings of how clients of their agencies have been affected by others' drinking. However, other than these proposed interviews, studies in the developing AHTO field have not included qualitative research with survey respondents adversely affected by others' drinking.

Based on the Australian 2008 survey data, according to parental carers, 12 per cent of children had been yelled at, criticised or verbally abused, left unsupervised or in an unsafe situation, physically hurt, subject to a child

Correspondence: Elizabeth Manton, Centre for Alcohol Policy Research, Turning Point, 54-62 Gertrude Street, Fitzroy, Victoria 3065, Australia. Telephone: +61 3 8413 8413; Fax: +61 39416 3420; E-mail: Elizabethm@turningpoint.org.au

Financial support: This work was supported by the Foundation for Alcohol Research and Education, an independent, charitable organization working to prevent the harmful use of alcohol in Australia: www.fare.org.au.

Keywords: Alcohol's harm to others, qualitative, children 
protection report, and/or had witnessed serious violence in the home at least once during the previous 12 months. However, in response to a more general question, 17 per cent reported that the drinking of other people (not necessarily a family member) had negatively affected their child or children in the past year; the extent of negative effect was identified as "a lot" by 3 per cent and "a little” by 14 per cent. To access more detailed information on harms experienced by children and families as a result of another person's drinking, we conducted follow-up qualitative research interviews with survey respondents caring for children who had been negatively affected in this way. A qualitative study such as this can help evaluate and interpret results from the principally quantitative study (Morgan, 1998). Our follow-up study was designed to access more detailed accounts of harm than is possible using survey research and also to enable us to better interpret survey results. The purpose of this paper is to use our research project as a case study to examine the potential contribution that qualitative components can make to future international AHTO work.

\section{Methods}

The 2008 AHTO survey was based on a national random sample of 2,649 Australians aged 18 years or over who were fluent in English (Laslett et al., 2010, p. 22). In 2011, a follow-up survey entailed re-interviewing 1,106 participants (Laslett et al., 2013). The resultant sample was Australia-wide, and included participants from a broad range of socioeconomic backgrounds. In the follow-up qualitative project, 20 in-depth semi-structured interviews were undertaken (Johnson, 2001). Potential participants were those who, in either the 2008 or 2011 AHTO survey, indicated that a child or children for whom they had responsibility had been harmed "a lot" or "a little" by someone else's drinking, and who also indicated that they were happy to be recontacted for future research interviews. The inclusion in the survey of this question about willingness to be recontacted was crucial to enabling this subsequent qualitative research project. Interviews were designed to elicit information about the nature of the impacts of drinking on children; the impacts of drinking on family functioning; the supports received or not received by the respondents; and demographic details. However, the interviews were also open-ended, in that interviewees were allowed as long as they liked to reply and emerging themes were probed further. Ethics approval for the project was granted through Eastern Health Research and Ethics Committee (project number E45/1112).

Because participants lived across Australia, the interviews were conducted by telephone. On initial contact, a script was read out to potential participants reminding them of their previous participation and their stated willingness to be recontacted for future research, and providing an overview of what the current interview entailed. The potential participants were always asked if this was a convenient time for them to talk, with the interviewer being mindful that the drinker who was harming the children might be present near the participant. At this stage, participants could agree to be interviewed immediately; agree to be interviewed, but at a more convenient time; request more information, in which case a Plain Language Statement (PLS) was sent to them and a recontact time was agreed; or refuse to be interviewed.

At the start of the interview, the participants were asked to provide verbal consent to participate. Interviews were audio recorded and professionally transcribed. Transcribed interviews were stored and analysed using NVivo9, a qualitative software package that enables thematic analysis of large amounts of text (Braun \& Clarke, 2006).

When completing the initial surveys, 21 respondents had answered "a lot" when asked how much harm children in their care had sustained and had also indicated that they were prepared to be recontacted. Of these prospective interviewees, we were able to interview 10 (eight females and two males). Eighty-seven had answered "a little" and indicated they were prepared to be recontacted. Of these, 10 were interviewed (seven females and three males). The order in which they were selected for contact was based on a randomly generated list of these 87 prospective interviewees. After preliminary analysis of all the interviews, it was decided not to access additional participants who had answered "a little," as no new themes were arising from analysis of these participants, and the harms experienced were usually much less severe than the harms recounted by those who answered "a lot," as identified in the first paragraph of the next section.

Sixteen of the 20 interviewees were the parents of children being harmed by another person's drinking. The remaining interviewees were grandparents who had a range of care arrangements for their grandchildren, often with shifting patterns of formal custody and informal caregiving arrangements.

The 15 female interviewees ranged in age from 28 to 70 , with 10 of them being aged between 40 and 60 . The five male interviewees ranged in age from 47 to 57 . Four of the women had a degree or diploma, three interviewees had completed secondary school, and half of the interviewees had not completed secondary school. Nearly three-quarters of the interviewees lived in major cities, while the rest were spread between regional centers and remote areas. Almost all the interviewees described themselves as coming from an AngloAustralian background. Relationship status is discussed in the text. No information was collected on income levels, as we felt that the themes we wished to discuss made interviews already potentially intrusive. Each interviewee has been given a pseudonym, and "A" or "B" has been added to the name to indicate whether the respondent had answered "a lot" or "a little," respectively. All interviewee quotes are from parents, except where a grandparent is separately identified.

The contributions of qualitative research outlined in this paper were identified through discussion between the authors, which occurred as part of the process of undertaking the study. Results and discussion sections are integrated in this paper to avoid duplication of text.

\section{Results and Discussion}

Harms that were identified by those answering "a lot" included being yelled at, criticised or verbally abused; being emotionally neglected; witnessing serious violence; 
witnessing physical conflict; witnessing verbal conflict; and being in an unsafe situation. Harms that were identified by those answering "a little" included witnessing drinking and witnessing inappropriate behavior. These harms are now discussed in more detail.

\section{Access to Detailed Stories}

In the 2008 AHTO survey, respondents with children reported that the most common harm experienced by the children was "being yelled at, criticised or verbally abused," which was reported by eight per cent of respondents. In our qualitative study, there were descriptions of the verbal abuse directed at children:

She'll be yelling and screaming and get in the f-ing, you know speaking like that, terrible language. [Child] has got in the car crying, the seven-year-old (BarbaraA, grandmother of children).

An associated theme arising from the interviews was "emotional neglect":

I lost my job and went through a depression and drank at home to the point where it did affect my ability to do as much as I wanted to do with them at times. ... I withdrew to a degree from family interaction (IsabelleA).

The literature on harms to children as a result of someone else's drinking found neglect, violence, or abuse, or exposure to these, to be the main harms both internationally (Holmila, Itäpuisto, \& Ilva, 2011; Mongan, Hope, \& Nelson, 2009; Orford, Velleman, Copello, Templeton, \& Ibanga, 2010; Velleman, Templeton, Reuber, Klein, \& Moesgen, 2008) and in Australia (Dawe et al., 2007; Gruenert, Ratnam, \& Tsantefski, 2004; Laslett et al., 2010). So while the emotional neglect theme had not been measured specifically in the Australian AHTO survey, it was not new.

The next most frequently observed harm in the survey was "witnessing serious violence," reported by three per cent of respondents (again, concurring with the literature). This also emerged in our qualitative study:

I suppose the impact happened when he actually one night was physically abusive to [their] mother and the three of them witnessed it. ... They'd been out to a party and something she said to him, stop drinking or something .... and when he got in the door he actually tried to strangle her (MargaretB, grandmother of children).

The wording of the survey question, with its emphasis on "serious" violence, left untapped the most common type of harm reported by the qualitative study interviewees, which was the children "witnessing conflict":

My son was seeing it all the time.

Interviewer: You trying to get him to stop drinking? Just generally we would fight all the time and the fact that he was seeing his father drink all the time. Interviewer: Was this physical fighting or verbal fighting? Physical and verbal (NarelleA).

"Children [being] left in an unsupervised or unsafe situation" was reported in the survey by three per cent of respondents. In our qualitative study, drink driving was reported by one woman, who described taking her children out of a car being driven by her drunk sister:
[My children] were in the car with [my sister] one day when she had been drinking and I followed her and stopped the car and asked them to get out and then took them myself. . . . I felt they were unsafe so I never let them go to her place (BelindaA).

This resonated with Connor and Casswell's (2009) finding in New Zealand that children injured in drink driving cases were usually in the same car as the drunk driver.

One per cent of the respondents in the AHTO survey responded in the affirmative to the question, "Were children physically hurt?" There were no instances reported in the interviews of children being physically hurt. It was, rather, the potential for physical harm to children which was reported:

[My son] used to wake up during the night sometimes and [my other son-the drinker] had knives at his throat (YvonneA).

These and other stories from our qualitative research provide nuanced and detailed explications of the kinds of events which triggered people to respond to survey questions about harms experienced by children as a result of another person's drinking.

\section{Clarifying the Validity and Meanings of Survey Measures}

One of the aims of our research was to identify whether there is any real difference between what respondents identify as "a little" and "a lot" of this kind of harm when they respond to AHTO survey questions, as discussed in the Introduction. In the discussion of specific harms in the preceding section, almost all the examples were drawn from the interviewees who had originally responded that their children were harmed "a lot." MargaretB, quoted above, had actually replied "a little"; however, her story might well have warranted a reply of "a lot." Further qualitative research could tease out how and why individuals and families living with violence may rate the severity of violence differently.

In our qualitative study, among children who were harmed "a little," the most common harm was that they had witnessed drinking and inappropriate behavior, rather than conflict. Examples of this behavior were tipsiness, being hard to talk to, inappropriate language, and being inconsistent with emotions:

She went out a few times and came back a little bit tipsy and that's all they noticed (MichaelB).

Often, it was easier for these respondents to control what behavior the children witnessed than it was for respondents whose children were affected "a lot," because the drinker was not in the immediate family:

He would always ring up on the phone and that's when I knew he was drunk and it was always hard to get off the phone. So that's when the kids knew, oh mum's friend. They used to refer to him as mum's drunk friend (LolaB).

In cases where the respondent answered "a lot," eight of the drinkers were parents of the child harmed, and one was a brother of the child. Only one drinker, an aunt, was identified as being in the extended family rather than the immediate family. In cases where the response was "a little," five of the drinkers were parents. One drinker, 
an uncle, was in the extended family, but more importantly, at least four of the drinkers were outside the extended family, either friends or strangers.

The results in this section imply that survey respondents who replied "a little" should be considered distinctly different from those who answered "a lot." As discussed above, out of 10 respondents who answered "a little," only one could reasonably have answered "a lot." For the most part, "a little" meant qualitatively less harm than "a lot." This distinction was also explored quantitatively in the 2011 AHTO survey, which asked respondents to rate their level of harm from 1 to 10 . This found that the mean score of the respondents who were harmed "a little" was 3.81, while the mean score of the respondents who were harmed “a lot” was 8.19 (Laslett et al., 2013).

\section{Identifying questions for inclusion in future population-based AHTO survey research}

A further methodological benefit of conducting qualitative AHTO studies is the capacity to identify questions for incorporation in future surveys. In the first section above, we identified "emotional neglect" and "witnessing conflict" as harms that were reported by interviewees. "Witnessing conflict" could be broken down into "witnessing physical conflict," "witnessing verbal conflict," and "witnessing both physical and verbal conflict." These four harms could be added to future AHTO surveys.

There was a further important finding from the qualitative study that was not examined in the AHTO survey. Marital disharmony and/or breakdown has previously been identified as one of the impacts on children when one parent is a problematic drinker (Templeton, Velleman, \& Russell, 2010; Zeitlin, 1994). In the current qualitative study, the main impact on the family of having a parent whose drinking was harming children was that the other parent was prepared to leave the relationship, a finding which applied for both men and women. In one case the threat of separation effected a change:

My husband was a binge drinker until my daughter was three. My son was five. . . . I got to the point where I told him that I didn't want them growing up with . . . an alcoholic father. . . . It was either the over-drinking or the kids. I was at the point where I was prepared to leave (IsabelleA).

Nonetheless, this finding should be viewed in the context of the limitations imposed by sampling for our qualitative research. The preparedness of prospective respondents to be interviewed for the qualitative study may have been related to their having reached a point of resolution, such as a separation, or to their being sufficiently distanced from the situation-for example, as a grandparent or a foster carer- to be able to discuss the issues.

Several prospective interviewees who had answered "a lot" indicated on first approach that they were interested in talking to the interviewer at a more convenient time, but on re-call at their nominated time decided not to proceed. One possible explanation was that the harmful drinker was inadvertently involved in the telephone exchange, and as the interviewer could not self-identify for ethical reasons, this led to an uneasy situation. Alternatively, the problem drinker may have been present in the background. While this can only be speculation, it could be that people who no longer lived with the drinker who was harming the children were more likely to feel able to participate in this interview. That is, the high incidence of marital separation observed may not reflect the situation of people in the general population in an intimate relationship with a drinker. Including questions in future AHTO survey research to assess the prevalence of alcohol-related relationship breakdown would offer people opportunity to respond to questions of this nature more privately, to determine whether this finding applies more generally.

\section{Contextualising survey findings}

A further useful contribution of qualitative studies is to contextualize findings by identifying how they are influenced by the design of a survey. This became evident during interviews when qualitative research participants reflected, not only on the identified problem drinker, but also on the impact that their own drinking or that of other family members might have on children in their care.

In families in the Australian AHTO where a problematic drinker was identified, this person was usually a male (Berends, Ferris, \& Laslett, 2012). In the current study, based on 20 interviewees drawn from that survey, this held true; three-quarters of the adults whose drinking affected the interviewees' children were men, usually the children's fathers. However, in the process of being interviewed, several women in such families revealed that their own drinking was also affecting their children. At first, SharonA's story was about the impact of her former partner's drinking on their baby son:

Interviewer: Can you tell me, is there anyone whose drinking has affected these children at any time? The oldest as a baby. The home was broken up from alcohol dependency . . . we had problems because of his dad's alcoholism.

Interviewer: When did you break up from your son's dad?

He was 11 months old (SharonA).

But on further probing, made possible through the openended interview technique, she revealed more about her own drinking history:

I was off medication [for depression] after I had my first son and I did go through a period, after I finished breastfeeding, of binge drinking, but that stopped about a year and a half [later] (SharonA).

What the interviewees indicate is that some women's lower (but still problematic for the children) level of drinking may have been undercounted in Australian AHTO survey research. This is due to the tendency within the survey to focus on a particular problem drinker known to the respondent, rather than attending to all the drinking that occurs in his or her social environment. The interviewees' own drinking may have also influenced their responses in unknown ways.

\section{Conclusions}

Our interviews enabled elicitation of detailed stories, and such accounts can be powerful mechanisms to influence policy makers and public opinion (Fitzgerald, 2000). In this research, the pervasive nature of the effect of a caregiver's alcohol use on some children, particularly those affected "a lot," was striking. The self-reflection inherent in the qualitative interview process encouraged discovery of additional dimensions of harm from others' 
drinking that had not been initially evident—such as the effect of the respondent's own drinking.

We also identified some methodological advantages of undertaking a follow-up qualitative study. Rhodes (2000, p. 29) has written that qualitative studies enable researchers to think about alcohol and drug use without the constraints imposed by a need to develop measurable options for survey respondents to choose from, based on established expectations of the nature of alcohol-related harms. In our research, contrasting existing specific questions with qualitative data clearly identified gaps in closed-response survey questions and new questions which could be asked, both of which will lead to future survey improvements. There existed the possibility of recall problems, especially from respondents who had answered "a lot" or "a little" in 2008 but not in 2011. However, only one interviewee who had answered "a little" in 2008 but not in 2011 was unable to recall what harms she had been referring to in 2008 .

A more conceptual contribution of our qualitative study has been in encouraging us to think of problematic alcohol use as embedded in families, relationships and social contexts, rather than just in individual problem drinkers. This was particularly apparent when some qualitative research participants discussed how children in their care were affected by the drinking of a range of adults. While space constraints prohibit our exploring this in detail, future AHTO surveys should be designed to capture the effects of multiple drinkers on respondents' lives.

It is inevitable that samples of people who agree to discuss harm from others' drinking with a researcher will be skewed in particular ways. Overall, for example, the survey respondents who identified children in their care as having experienced "a little" harm identified trauma of greater severity than did our qualitative interview participants. The small sample size of our qualitative study meant that we were unlikely to capture the range of harm experienced. Nonetheless, accessing participants from a broad survey sample that reflects the general population is likely to result in a study sample with more diverse experiences, less biased towards the very severe end of the spectrum, than one which is recruited via health or welfare programs.

The approach to AHTO research we have described here incorporates both the persuasive power of wholepopulation survey research and the nuanced understanding provided through interpretation of in-depth qualitative interviews. It enables us to present more comprehensive information about the nature and extent of AHTO.

\section{References}

Berends, L., Ferris, J., \& Laslett, A.-M. (2012). A problematic drinker in the family: Variations in the level of negative impact experienced by sex, relationship and living status. Addiction Research and Theory, 20, 300-306.

Braun, V., \& Clarke, V. (2006). Using thematic analysis in psychology. Qualitative Research in Psychology, 3, 77-101.
Connor, J., \& Casswell, S. (2009). The burden of road trauma due to other people's drinking. Accident Analysis and Prevention, 41, 1099-1103.

Dawe, S., Fry, S., Best, D., Moss, D., Atkinson, J., Evans, C., . . . Hartnett, P. (2007). Drug use in the family: Impacts and implications for children ANCD Research Paper. Canberra, Australia: Australian National Council on Drugs.

Fitzgerald, J. (2000). Selling stories to the masses: Qualitative research and Australian drug policy. In G. Greenwood \& K. Robertson (Eds.), Understanding and responding to illicit drug use: The role of qualitative research (pp. 309-316). Luxembourg, Europe: European Monitoring Centre for Drugs and Drug Addiction.

Giesbrecht, N., Cukier, S., \& Steeves, D. (2010). Collateral damage from alcohol: Implications of 'second-hand effects of drinking' for populations and health priorities. Addiction, 105, 1323-1325.

Gruenert, S., Ratnam, S., \& Tsantefski, M. (2004). The Nobody's Clients Project: Identifying and addressing the needs of children with substance dependent parents. Victoria, Australia: Odyssey House.

Holmila, M. J., Itäpuisto, M., \& Ilva, M. (2011). Invisible victims or competent agents: Opinions and ways of coping among children aged 12-18 years with problem drinking parents. Drugs: Education, Prevention, and Policy, 18, 179-186.

Johnson, J. M. (2001). In-depth interviewing. In J. F. Gubrium \& J. A. Holstein (Eds.), Handbook of interview research: Context and method (pp. 103120). Thousand Oaks, CA, United States: Sage Publications.

Laslett, A.-M., Callinen, S., Mugavin, J., Jiang, H., Livingston, M., \& Room, R. (2013). Continuity and change in harm from others' drinking: Experiences of Australian adults in 2008 and 2011. Canberra, Australia: FARE Centre for Alcohol Policy Research, Turning Point Alcohol and Drug Centre.

Laslett, A.-M., Catalano, P., Chikritzhs, Y., Dale, C., Doran, C., Ferris, J., . . . Wilkinson, C. (2010). The range and magnitude of alcohol's harm to others. Fitzroy, Victoria, Australia: AER Centre for Alcohol Policy Research, Turning Point Alcohol and Drug Centre, Eastern Health.

Mongan, D., Hope, A., \& Nelson, M. (2009). Social consequences of harmful use of alcohol in Ireland. HRB Overview Series 9. Dublin, Ireland: Health Research Board.

Morgan, D. L. (1998). Practical strategies for combining qualitative and quantitative methods: Applications to health research. Qualitative Health Research, 8, 362-376. doi:10.1177/104973239800800307

Orford, J., Velleman, R., Copello, A., Templeton, L., \& Ibanga, A. (2010). The experiences of affected family members: A summary of two decades of qualitative research. Drugs: Education, Prevention, and Policy, 17(s1), 44-62.

Rhodes, T. (2000). The multiple roles of qualitative research in understanding and responding to illicit drug use. In G. Greenwood \& K. Robertson (Eds.), Understanding and responding to illicit drug use: The role of qualitative research (pp. 21-36). Luxembourg, Europe: European Monitoring Centre for Drugs and Drug Addiction. 
Templeton, L., Velleman, R., \& Russell, C. (2010). Psychological interventions with families of alcohol misusers: A systematic review. Addiction Research \& Theory, 18, 616-648.

Velleman, R., Templeton, L., Reuber, D., Klein, M., \& Moesgen, D. (2008). Domestic abuse experienced by young people living in families with alcohol problems: Results from a cross-European study. Child Abuse Review, 17, 387-409.

World Health Organization. (2010). Global strategy to reduce the harmful effects of alcohol. Geneva, Switzerland: Author.

Zeitlin, H. (1994). Children with alcohol misusing parents. British Medical Bulletin, 50, 139-151. 